\title{
Consulta de enfermagem: avaliação da adesão ao autocuidado dos pacientes submetidos à radioterapia
}

\author{
Nursing consultation: evaluation of adherence to self-care in patients undergoing radiotherapy \\ Consulta de enfermería: evaluación de la adhesión al autocuidado de los pacientes sometidos a \\ la radioterapia
}

\author{
Karla Biancha Silva de Andrade; Anna Clara Lima Francz"I Marianne dos Santos Grellmannn; \\ Priscila Cortez Belchiorr ; Josiana Araujo de Oliveira ${ }^{V}$; Dayse do Nascimento Wassita ${ }^{\text {VI }}$
}

\begin{abstract}
RESUMO: Esta pesquisa objetivou avaliar a adesão dos pacientes às orientações fornecidas na consulta de enfermagem em radioterapia. Trata-se de um estudo descritivo, com abordagem quantitativa, realizado em um Centro Universitário de Controle do Câncer no Estado do Rio de Janeiro. Foram avaliados 37 pacientes submetidos à radioterapia, através de questionário próprio, contendo 16 itens relacionados ao perfil e adesão dos pacientes aos cuidados orientados, aplicado em maio de 2012. Os resultados mostraram que a maioria dos entrevistados foi do sexo masculino (62\%), com faixa etária predominante de 58-67 anos (38\%), a maior parte tratou de tumores na cabeça e pescoço $(51 \%), 90 \%$ referiram aderir às orientações de autocuidado e $54 \%$ não desenvolveram radiodermites. Concluiu-se que a consulta de enfermagem é importante no cuidado aos pacientes que se submetem à radioterapia e pode influir na adesão ao tratamento e no esclarecimento de dúvidas sobre o plano terapêutico.
\end{abstract}

Palavras-Chave: Consulta de enfermagem; radioterapia; autocuidado; adesão.

ABSTRACT: This research aimed at evaluating patients' adhesion to directions received in nursing consultation in radiotherapy. This is a descriptive study with quantitative approach conducted at a University Center for Cancer Control in the State of Rio de Janeiro, RJ, Brazil. Thirty-seven (37) patients undergoing radiotherapy were evaluated through a questionnaire, applied in May, 2012 and containing 16 profile and adhesion-related items concerning guided care. Results showed the majority of patients was males $(62 \%)$, aging from $58-67$ years old, $(38 \%)$ undergoing treatment for head and neck tumors $(51 \%)$. Ninety per cent $(90 \%)$ reported adhesion to self-care directions and 54\% did not develop radiodermatitis. Conclusions showed the relevance of nursing consultation in care to patients undergoing radiotherapy as to their adhesion to treatment and clarification of doubts on their therapy.

Keywords: Nursing consultation; radiotherapy; self-care; adhesion.

RESUMEN: Esta investigación objetivó evaluar la adhesión de los pacientes a las orientaciones fornecidas en la consulta de enfermería en radioterapia. Se trata de un estudio descriptivo, con enfoque cuantitativo, realizado en un Centro Universitario de Control del Cáncer en el Estado de Rio de Janeiro - Brasil. Fueron evaluados 37 pacientes sometidos a la radioterapia por medio de un cuestionario propio, conteniendo 16 preguntas relacionadas al perfil y adhesión de los pacientes a los cuidados orientados, aplicado en mayo de 2012. Los resultados revelaron que la mayoría de los entrevistados fue del sexo masculino $(62 \%)$, con franja etaria predominante de 58 - 67 años (38\%), la mayor parte trató de tumores en la cabeza y cuello $(51 \%)$, $90 \%$ declararon adherir a las orientaciones de autocuidado y $54 \%$ no desarrollaron radiodermites. Se concluyó que la consulta de enfermería es importante en el cuidado a los pacientes sometidos a la radioterapia y puede influir en la adhesión al tratamiento y en el esclarecimiento de dudas sobre el plan terapéutico.

Palabras Clave: Consulta de enfermería; radioterapia; autocuidado; adhesión.

\section{INTRODUÇÃO}

O câncer é uma doença crônica e constitui um contínuo problema de saúde pública em todo o mundo. A cada ano, o número de casos diagnosticados cresce. Para 2014 e 2015, as estimativas do Instituto Nacional do Câncer (INCA) apontam para a ocorrência de aproximadamente 576 mil casos novos de câncer no Brasil, reforçando a magnitude do problema ${ }^{1}$.
Diante destes dados, é oportuno desenvolver estratégias de prevenção e controle da doença, acesso ao diagnóstico e tratamento em tempo adequado, bem como, um cuidado seguro que minimize os efeitos indesejáveis provenientes do plano terapêutico.

Entre os tratamentos do câncer, a radioterapia é utilizada em larga escala, podendo ter a finalidade

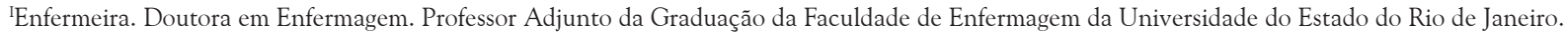
Enfermeira do Instituto Nacional do Câncer. Rio de Janeiro, Brasil. E-mail: k.biancha@ig.com.br.

IIEnfermeira. Graduada pela Universidade do Estado do Rio de Janeiro. Brasil. E-mail: annafrancz@yahoo.com.br

IIIEnfermeira. Pós-Graduanda em Oncologia pela Universidade Federal Fluminense. Rio de Janeiro, Brasil. E-mail: mari.grell@hotmail.com

IVEnfermeira. Graduada pela Universidade do Estado do Rio de Janeiro. Brasil. E-mail: pribelchior@hotmail.com

${ }^{v}$ Enfermeira. Mestranda em Enfermagem pela Universidade do Estado do Rio de Janeiro. Pós-Graduada em Terapia Intensiva (Residência). Professora

Substituta da Faculdade de Enfermagem da Universidade do Estado do Rio de Janeiro. Brasil. E-mail: josianaoliveira@yahoo.com.br

VIEnfermeira. Especialista em Enfermagem Oncológica pelo Instituto Nacional do Câncer. Chefe de Enfermagem do Serviço de Radioterapia do Hospital

Universitário Pedro Ernesto. Rio de Janeiro, Brasil. E-mail: 
curativa ou paliativa, nas modalidades de teleterapia e braquiterapia. Entretanto, apesar de seus resultados desejados, os indivíduos em contato com a radiação podem desenvolver manifestações de toxicidade, como as radiodermites e mucosites ${ }^{2,3}$. As reações iniciais da pele, após a radioterapia, são geralmente moderadas, porém, mais de $90 \%$ dos pacientes tratados com radioterapia desenvolvem reações cutâneas ${ }^{2}$.

Dessa forma, medidas de prevenção devem ser desenvolvidas a fim de minimizar a radiotoxicidade aguda durante o tratamento. A consulta de enfermagem torna-se fundamental nesse momento, sendo ferramenta essencial para a qualidade de vida do paciente e condição favorável para se ofertar um cuidado seguro ${ }^{4}$.

O protocolo do INCA sistematiza as orientações dadas durante a consulta de enfermagem, através de um guia de autocuidado com a pele irradiada, para minimizar a exacerbação dos danos provocados pela radiação utilizada durante o tratamento, mesmo considerando sua limitação para impedir o desenvolvimento de tais reações ${ }^{5,6}$.

Entre as orientações de autocuidado contidas nesse protocolo e realizadas pela enfermeira, pode-se citar: usar creme hidratante à base de aloe vera, evitar jato de água diretamente na pele irradiada e depilação com lâmina ou cera, incentivar a ingestão de dois litros de líquidos por dia e recomendar a não exposição da pele ao sol durante o tratamento ${ }^{5,6}$.

Tais orientações devem compor o plano terapêutico que, ainda, deve ressaltar a importância do comparecimento às consultas, as mudanças de hábitos de vida ao longo do tratamento e o esclarecimento de dúvidas que forem surgindo durante este período. Nesse sentido, vale destacar como questionamento, se a consulta de enfermagem pode contribuir na minimização das complicações relacionadas à radioterapia nos pacientes oncológicos que se submetem ao tratamento.

A consulta de enfermagem elucida dúvidas comuns e isoladas de pacientes que iniciam o processo terapêutico, constituindo-se como momento oportuno para o melhor entendimento do paciente sobre a doença e a importância da adesão às sessões de radioterapia $^{4-7}$. Dessa forma o presente estudo objetivou avaliar a adesão dos pacientes às orientações realizadas na consulta de enfermagem em radioterapia.

\section{REVISÃO DE LITERATURA}

A radioterapia utiliza radiação ionizante, com energia suficiente para ionizar moléculas através da liberação de elétrons da estrutura atômica, como os raios X e partículas beta e alfa. Os radioisótopos são utilizados em doses elevadas, visando justamente o efeito deletério da radioatividade sobre determinados tecidos, objetivando alcançar um índice terapêutico favorável e levando as células malignas a perderem a sua clonogenicidade ${ }^{2,7}$.
A pele é um órgão caracterizado por possuir células com ciclo de rápida divisão celular, um fator importante da radiossensibilidade, e, por esse motivo, é o primeiro tecido a manifestar reações adversas à radiação ionizante ${ }^{5}$. Sendo assim, as radiodermites são lesões cutâneas consideradas eventos adversos e podem acometer aproximadamente $95 \%$ dos pacientes tratados com radioterapia. As reações de pele mais comuns são: pele ressecada, prurido e eritema após três semanas da finalização das radiações ${ }^{2,5,8}$.

O protocolo do INCA tem sido amplamente utilizado por hospitais e serviços de oncologia, de maneira a potencializar a eficácia das ações preventivas ${ }^{5-7}$. Esse protocolo preconiza, ainda, a avaliação de parâmetros de localização tumoral, de doses e energia específica que interferem na toxicidade aguda da pele e mucosa oral, e a orientação de práticas de autocuidado, com o objetivo de prevenir complicações e/ou minimizar os efeitos colaterais inevitáveis ${ }^{2,5,8}$.

De acordo com a Resolução COFEN noํㅜ 159/1993, a consulta de enfermagem é uma atividade privativa do enfermeiro e utiliza componentes do método científico para identificar situações de saúde/doença, prescrever e colocar em prática ações de enfermagem que contribuam para a promoção da saúde, prevenção e proteção de agravos, recuperação e reabilitação do indivíduo, família e comunidade 9 .

Além disto, a Resolução COFEN no 211/1998 regulamenta a atuação dos profissionais de enfermagem nos serviços de Radioterapia, Medicina Nuclear e Imagem ${ }^{10}$. Em consonância com essas resoluções, o enfermeiro deve participar de protocolos terapêuticos de enfermagem, promover e difundir medidas de saúde preventivas e curativas, utilizando-se da consulta de enfermagem para formular e aplicar manuais explicativos através da educação aos pacientes e familiares, adequando-os às suas realidades sociais ${ }^{5-7,10}$.

\section{Metodologia}

Trata-se de um estudo descritivo com abordagem quantitativa em função do objeto selecionado. Um estudo do tipo descritivo tem a finalidade de estudar as características de um grupo, levantamento de opiniões, atitudes, crenças de uma população ou identificação das relações entre as variáveis ${ }^{11,12}$.

A pesquisa foi realizada no Centro Universitário de Controle do Câncer, serviço de referência em radioterapia de um hospital universitário situado no Município do Rio de Janeiro, que oferece tratamento radioterápico. Tal serviço abriga a Unidade Docente Assistencia (UDA) de Radioterapia, o Ambulatório de Cuidados Paliativos e parte do Serviço de Radiologia e Diagnóstico por Imagem. Segundo informações fornecidas pela unidade, é atendida mensalmente uma média de 50 pacientes. 
Participaram desta pesquisa os pacientes em tratamento radioterápico para dois grupamentos de especialidades: câncer de cabeça e pescoço, mama, pelve feminina, pelve masculina e, tórax e abdômen, os quais foram submetidos a quatro e três consultas de enfermagem, respectivamente, nos diversos momentos que norteiam esse tratamento.

$O$ instrumento de coleta de dados foi um questionário próprio, formulado com 16 questões fechadas, dividido em duas partes: a primeira parte, com cinco questões sobre o perfil do paciente incluindo as variáveis sociodemográficas (idade, sexo, grau de instrução e renda); e a segunda parte, com 11 questões acerca de sua adesão aos cuidados orientados durante a consulta de enfermagem, contendo as variáveis: desenvolvimento de radiodermites, adesão às orientações de autocuidado para a prevenção de radiodermites e localização do tumor.

Foram elencados cinco itens referentes aos cuidados que devem ser realizados: os cuidados gerais com a pele, o uso de hidratante de aloe vera, a manutenção das unhas aparadas e limpas, a ingesta de 2 litros de água por dia e o uso de chá de camomila sobre a pele. Consideraram-se, também, cinco itens sobre ações que devem ser evitadas: a força de jato de água na pele, o uso de lâmina ou cera, o uso de roupas escuras e de materiais sintéticos, a exposição da pele ao sol e o esforço físico.

Os critérios para inclusão dos sujeitos na pesquisa foram: consentimento de participação, ser alfabetizado, ter mais de 18 anos de idade e estar agendado para a penúltima ou última consulta de enfermagem no dia da aplicação do questionário. $\bigcirc$ último critério justificou-se pela maior possibilidade do paciente apresentar efeitos adversos à radioterapia e o maior período de relação entre enfermeiro-paciente. Já os de exclusão foram: analfabetismo, dor no momento da aplicação do questionário e ser portador de deficiência auditiva, visual e/ou motora.

O projeto foi submetido à Comissão de Ética em Pesquisa ${ }^{13}$ da Sub-Reitoria de Pós-Graduação e Pesquisa da Universidade do Estado do Rio de Janeiro e aprovado sob o parecer $n^{\circ}$ 006.3.2012. A coleta de dados ocorreu em maio de 2012, sendo iniciada somente após a aprovação da chefia da unidade e a assinatura do Termo de Consentimento Livre e Esclarecido pelos pacientes.

Os dados foram tabulados e analisados por meio da frequência simples. As informações obtidas geraram um banco de dados, o qual embasou a criação de tabelas, através do software MS-Excel versão 2007.

\section{Resultados e Discussão}

Participaram do estudo 37 indivíduos. A análise das informações sociodemográficas demonstrou que, deste conjunto, 22 (62\%) são do sexo masculino; que a faixa etária prevalente foi a de 58 a 67 anos, com 14
(38\%) participantes; 16 (43\%) recebem de um a dois salários mínimos, seguido de 12 (32\%) que recebem até um salário mínimo. Cerca de um terço dos participantes possui ensino fundamental completo (11), seguidos dos 9 (24\%) que possuem ensino fundamental incompleto, como demonstrado na Tabela 1.

TABELA 1: Perfil sociodemográfico dos pacientes avaliados . Rio de Janeiro, Centro Universitário de Controle do Câncer, 2012. $(\mathrm{N}=37)$

\begin{tabular}{lcc}
\hline \multicolumn{1}{c}{ Variáveis } & f & $\%$ \\
\hline Sexo & 23 & 62 \\
Masculino & 14 & 38 \\
Feminino & & \\
Idade & 5 & 14 \\
Menor de 48 anos & 9 & 24 \\
De 48 a 57 & 14 & 38 \\
De 58 a 67 & 9 & 24 \\
Acima de 67 & & \\
Grau de Instrução & 9 & 24 \\
Ensino fundamental incompleto & 11 & 30 \\
Ensino fundamental completo & 17 & 46 \\
Outros & & \\
Renda & 12 & 32 \\
Até 1 salário mínimo & 16 & 43 \\
De 1 a 2 salários mínimos & 94 \\
Outros & 11 & 24 \\
Localização do tumor & 4 & 10 \\
Cabeça e pescoço & 31 \\
Pelve masculina & & \\
Mama & & \\
Pelve feminina, tórax e abdomen & & \\
\hline & & \\
\hline
\end{tabular}

Os resultados divergem da literatura, em relação à prevalência do câncer de acordo com sexo, pois estatísticas nacionais apontam que não há diferença significativa na prevalência e incidência entre os sexos. As diferenças se dão, basicamente, através da localização tumoral e letalidade de cada tipo de câncer. As faixas etárias predominantes encontradas têm estreita relação com as localizações tumorais dos participantes, como mama, cabeça e pescoço e próstata ${ }^{1,14}$.

grau de instrução está associado ao maior conhecimento sobre o câncer, e a renda mais elevada pode influenciar a história da patologia do paciente, já que o câncer é mais prevalente entre os grupos de maior exclusão social, pois estes se submetem a maiores fatores de risco de adoecimento e morte ${ }^{1,14,15}$. Assim, há relação entre a renda e localizações tumorais ligados a hábitos de vida, como o câncer de boca e do colo uterino, mais frequente entre adultos jovens de baixa renda e escolaridade ${ }^{14,15}$. 
Em relação à localização do tumor, 19(51\%) trataram tumores de cabeça e pescoço, seguidos de 11(30\%) com tumor de pelve masculina e 4(11\%) de mama.

Os tumores que compõem o rol cabeça e pescoço, como laringe e esôfago, têm grande incidência na população, mas não superam a ocorrência de câncer de mama e próstata, por exemplo. O câncer da próstata, patologia da pelve masculina, foi considerado o segundo tipo de câncer mais frequente em homens. Já o câncer da mama é o tipo de câncer que mais acomete as mulheres em todo o mundo, tanto em países em desenvolvimento quanto em países desenvolvidos ${ }^{1,14,15}$.

A avaliação da adesão às orientações de autocuidado, fornecidas durante as consultas de enfermagem, demonstrou que 33(90\%) participantes relataram ter aderido às recomendações e 20(54\%) referiram não ter desenvolvido radiodermites durante o tratamento radioterápico.

Observa-se que a utilização da radioterapia para o tratamento do câncer tem se mostrado um efetivo adjuvante na regressão dos tumores ${ }^{15,16}$. Apesar do avanço, efeitos adversos ainda são observados durante e após o tratamento radioterápico. Um dos efeitos mais comuns são as reações agudas de pele, as quais podem variar de um leve eritema e prurido até a necrose tecidual, passando por descamação seca ou úmida ${ }^{2,4-6,8}$.

Nesse sentido, as medidas de prevenção precisam ser prescritas durante a consulta de enfermagem, com intuito de diminuir a radiotoxicidade $2,4-6,8,17,18$.

A distribuição dos cuidados adotados pelos clientes está descrita na Tabela 2.

Em relação aos cuidados com a pele, 36(97\%) afirmaram ter realizado tais cuidados na região irradiada, 30 (81\%) informaram ter ingerido dois litros de líquido por dia durante o tratamento, 36(97\%) utilizaram o hidratante de ecossoma de aloe vera, 34 (92\%) fizeram uso do chá de camomila para suavizar os efeitos irritativos na pele. Além disso, evidenciou-se que a maior parte optou por manter as unhas aparadas e limpas, perfazendo 31(84\%) participantes.

A necessidade basal de água para o adulto depende das perdas de água sensíveis (urinárias) e insensíveis, e varia de 1.250 a $3.000 \mathrm{ml} /$ dia, dependendo da superfície corporal, quantidade de massa celular, idade e sexo. Ademais, deve-se fazer a correção frente a estados clínicos de perda ou retenção. Desse modo, faz-se necessário o estímulo para o monitoramento da ingestão hídrica, visando à hidratação adequada para a eficácia e diminuição dos efeitos tóxicos durante o tratamento ${ }^{17-19}$.

Apesar de a maioria afirmar a ingesta de 2 litros de líquidos por dia, 5(16\%) não possuem essa prática e $1(3 \%)$ não lembrava se assim procedia. Esta informação instrumentaliza o enfermeiro para que, durante a consulta de enfermagem, dê maior ênfase quanto à ingesta de líquidos, hábito esse que deveria ser comum a qualquer indivíduo, independente de sua patologia ${ }^{19,20}$.

Na unidade em que foi realizada a pesquisa, são ofertados o hidratante de ecossoma de aloe vera, que deve ser utilizado após a sessão de radioterapia, e o sabonete hidratante, que deve ser usado para lavar a região irradiada. Com isso, observou-se neste estudo maior adesão em relação aos cuidados relacionados à utilização desses materiais, visto que esses são entregues desde a primeira consulta.

Diante da eficácia dos cuidados preventivos observada, diversos seviços adotaram produtos ou curativos que, aplicados na área irradiada, visam impedir o aumento do grau de radiodermites, reduzindo o desconforto do paciente e o tempo de interrupção do tratamento radioterápico. Esse tratamento tópico implica a aplicação de compressa de água e chá de camomila, loção à base de ácidos graxos essenciais (AGE) e aloe vera, para amenizar os efeitos da radiação na pele 2,4-6,20,21.

Apesar da insuficiência de estudos científicos comprovando a eficácia do uso da camomila, as unidades de tratamento de oncologia têm observado bons resultados, auxiliando na redução de danos e hidratação da pele, graças ao seu efeito emoliente $e^{6,20,21}$. Neste estudo, verificou-se que a adesão ao método fitoterápico foi expressiva (92\% usam o chá de camomila), seja pelo baixo custo, seja pelas crenças e valores, seja pela eficácia que desse método.

Outro cuidado muito importante é a manutenção das unhas limpas e aparadas, o que evita a lesão cutânea e a infecção no local de prurido, já que o paciente, ao coçá-lo, pode favorer as radiodermites,

TABELA 2: Relação dos cuidados que devem ser realizados x adesão dos participantes. Rio de Janeiro, Centro Universitário de Controle do Câncer, 2012. (N=37)

\begin{tabular}{lcccccc}
\hline \multicolumn{1}{c}{ Variáveis } & \multicolumn{2}{c}{ Sim } & \multicolumn{2}{c}{ Não } & \multicolumn{2}{c}{ Não lembra } \\
\cline { 2 - 7 } & $\mathrm{f}$ & $\%$ & $\mathrm{f}$ & $\%$ & $\mathbf{f}$ & $\%$ \\
\hline Cuidados gerais com a pele & 36 & 97 & 1 & 3 & - & - \\
Ingetão de 2 litros de líquido & 30 & 81 & 6 & 16 & 1 & 3 \\
Uso de hidratante de aloe vera & 36 & 97 & 1 & 3 & - & - \\
Uso de chá de Camomila & 34 & 92 & 3 & 8 & - & - \\
Manutenção das unhas aparadas e limpas & 31 & 84 & 6 & 16 & - & - \\
\hline
\end{tabular}


uma vez que o paciente oncológico sofre de imunossupressão e de sensibilidade cutânea devido à radioterapia $^{5,6,8,17,18}$.

Na consulta de enfermagem, além de orientações para o cuidado com a região irradiada, prevenindo o surgimento de radiodermites, também são fornecidas orientações de ações que devem ser evitadas durante o tratamento. Constatou-se que $32(84 \%)$ pacientes referem manter esses cuidados de fundamental importância, como demonstrado na Tabela 3, que descreve a relação entre as ações serem evitadas para prevenir complicações com a correspondente adesão dos participantes.

No conjunto desta pesquisa, 29(78\%) relataram evitar esforço físico. A fadiga é o mais frequente sintoma vivenciado por pacientes com câncer e sua mensuração e avaliação cuidadosas são decisivas para aferir a eficácia das estratégias de intervenção junto ao paciente e sua família. A fadiga é definida como uma sensação multidimensional, multicausal e similar à dor em sua complexidade. Por isso torna-se essencial evitar o esforço físico durante o tratamento, orientando a escolha pelo repouso e relaxamento ${ }^{17,21}$.

Além da fadiga, indisposição e anorexia são também verificadas. Estes sintomas podem ser secundários às substâncias liberadas quando se clivam as células tumorais. Os efeitos são temporários e diminuem com a interrupção do tratamento ${ }^{18,22,23}$.

Quanto a evitar jatos de água e o uso de roupas escuras e de material sintético, 30(81\%) participantes relataram aderir a tais medidas preventivas, bem como 35(97\%) evitaram o uso de lâminas ou cera e a exposição ao sol. Tais cuidados favorecem a manutenção da integridade cutânea por não causar atrito, arranhaduras ou trauma $4,6,8,17,18,20$.

Apesar de os resultados da pesquisa terem demonstrado um alto grau de adesão às orientações preventivas, observou-se que ainda predominou um expressivo índice de radiodermites. Isto levou à reflexão sobre alguns pressupostos, principalmente no que tange à confirmação da adesão dos pacientes ao tratamento preventivo proposto. É fato que não se pode confirmar que o paciente siga corretamente as orientações fornecidas, apenas através de suas respos- tas, uma vez que ele pode se sentir receoso em revelar o não cumprimento das mesmas, principalmente por terem sido orientados durante a consulta ${ }^{24,25}$.

A consulta de enfermagem aos pacientes e aos que estão envolvidos com sua doença deve considerar a história de vida de cada individuo, o impacto da doença e do seu tratamento na sua realidade. Assim, o plano de cuidados deve levar em consideração, além de outros aspectos, a realidade social dos sujeitos nessa condição $\mathrm{O}^{4,6,7,9,23-25}$.

Outro pressuposto é o temor de que, caso respondam algo que não esteja dentro do que foi orientado, possam ser tratados de maneira diferente, mesmo que este não cumprimento seja decorrente de alguma dificuldade individual, envolvendo recursos financeiros, por exemplo.

Nesse contexto, auxiliar o paciente a entender o processo em que está inserido é torna-lo capaz de enfrentar este momento de sua vida, apoiando-o para as possíveis complicações. Uma comunicação mais efetiva entre a equipe e o paciente poderá favorecer a adesão ao tratamento e aos cuidados a serem implementados, bem como, contribuir para minimizar os riscos da estratégia terapêutica ${ }^{4,6,24-26}$.

\section{Conclusão}

Os resultados da pesquisa proporcionaram uma análise sobre a consulta de enfermagem realizada na unidade de radioterapia, com vistas ao cumprimento de protocolos preconizados pelo Ministério da Saúde, seguindo um padrão de qualidade o qual pode influenciar diretamente na adesão às orientações de autocuidado.

É importante que o paciente compreenda os princípios de cada tratamento e a forma como eles se relacionam entre si. Isto possibilita o entendimento dos objetivos do plano terapêutico para a melhoria da qualidade de vida do indivíduo com doença oncológica.

O enfermeiro deve olhar o paciente na sua integralidade, considerando o aspecto socioeconômico, uma vez que tais aspectos podem influenciar a sua adesão às orientações de autocuidado durante o tratamento.

TABELA 3: Ações que devem ser evitadas na região da pele irradiada $X$ adesão dos participantes. Rio de Janeiro, Centro Universitário de Controle do Câncer, 2012. (N=37)

\begin{tabular}{|c|c|c|c|c|c|c|}
\hline \multirow[t]{2}{*}{ Variáves } & \multicolumn{2}{|c|}{ Sim } & \multicolumn{2}{|c|}{ Não } & \multicolumn{2}{|c|}{ Não lembra } \\
\hline & $f$ & $\%$ & $\mathrm{f}$ & $\%$ & $f$ & $\%$ \\
\hline Evitaram força de jato de água & 30 & 81 & 6 & 16 & 1 & 3 \\
\hline Evitaram utilizar lâmina ou cera & 36 & 97 & 1 & 3 & - & - \\
\hline Evitaram utilizar roupas escuras e de materiais sintéticos & 30 & 81 & 7 & 19 & - & - \\
\hline Evitaram expor a pele ao sol & 36 & 97 & 1 & 3 & - & - \\
\hline Evitaram esforço físico & 29 & 78 & 7 & 19 & 1 & 3 \\
\hline
\end{tabular}


Em relação ao tratamento radioterápico, o desenvolvimento de radiodermites é uma complicação adversa esperada, devido à toxicidade conferida pela radiação. Apesar de a maioria dos participantes afirmar ter aderido às orientações, a incidência de radiodermites ainda foi elevada. Concomitante a isso, a localização tumoral em cabeça e pescoço, por ser uma das regiões mais sensíveis à radiação acarreta, naturalmente, maior predominância dessas lesões.

Para tanto, a equipe de enfermagem, mais especificamente a enfermeira, deve propiciar um momento favorável para orientar ações de prevenção e solucionar dúvidas pertinentes.

A pesquisa abordou questões que perpassam por alguns aspectos em relação à adesão às orientações realizadas durante a consulta de enfermagem. Esses aspectos abrangem desde o envolvimento do paciente com o tratamento até o seu entendimento sobre a real necessidade da realização de práticas de autocuidado para reduzir os danos inerentes à radioterapia. Entendeu-se que, para se traçar um plano de cuidados aos pacientes nessa condição, é necessário que o enfermeiro leve em consideração como eles e seus cuidadores estão vivenciando o fenômeno estar doente. Para tal, é indispensável que haja integração entre pacientes, cuidadores e enfermeiros.

Um dos vieses da pesquisa foi a incerteza da veracidade das respostas, já que o estudo não acompanhou o decorrer do tratamento. O que fora respondido pelos participantes pode não traduzir o que verdadeiramente foi realizado. Outra limitação é que a grande parte deles possui baixa renda e baixa escolaridade, podendo influenciar a qualidade das respostas, por falta de um verdadeiro entendimento do processo.

É preciso estimular o desenvolvimento de novos estudos relacionados à temática, com outras metodologias que permitam explorar de forma mais cuidadosa os fatores que determinam a adesão ao autocuidado, contribuindo para a elucidação dessas lacunas.

\section{REFERÊNCIAS}

1.Instituto Nacional de Câncer José Alencar Gomes da Silva (Br). Estimativa 2014: incidência de câncer no Brasil. Rio de Janeiro: INCA; 2013.

2.Pires AMT, Segreto RA, Segreto HRC. Avaliação das reações agudas da pele e seus fatores de risco em pacientes com câncer de mama submetidas à radioterapia. Rev Latino-Am Enfermagem 2008; 16: 844-9.

3.Frigato $S$, Hoga LAK. Assistência à mulher com câncer de colo uterino: o papel da enfermagem. Revista Brasileira de Cancerologia 2003; 4(49): 209-14.

4.Araújo CRG, Rosas AMMTF. A consulta de enfermagem para clientes e seus cuidadores no setor de radioterapia de hospital universitário. Rev enferm UERJ.
2008; [citado em 29 abr. 2014] 16: 364-9. Disponível em: http://www.facenf.uerj.br/v16n3/v16n3a11.pdf. 5.Guedes MTS, Blecha FP. Tratamento de radiodermatite no cliente oncológico: subsídios para intervenções de enfermagem. Revista Brasileira de Cancerologia. 2006; 2(52):151-63.

6.Instituto Nacional de Câncer José Alencar Gomes da Silva $(\mathrm{Br})$. Ações de enfermagem para o controle do câncer: uma proposta de integração ensino-serviço. $3^{a}$ ed. Rio de Janeiro: INCA; 2008.

7.Araújo CRG, Rosas AMMTF. O papel da equipe de enfermagem no setor de radioterapia: uma contribuição para a equipe multidisciplinar. Revista Brasileira de Cancerologia. 2008; 3(54):231-7.

8.Naylor W, Mallet J. Management of acute radiotherapy induced skin reactions: a literature review. Eur J of Oncol Nurs. 2001; 4:221-3.

9.Conselho Federal de Enfermagem (Br). Resolução n 159/1993. Dispõe sobre a consulta de enfermagem. Rio de Janeiro: COFEN; 1993.

10.Conselho Federal de Enfermagem (Br). Resolução $n^{\circ}$ 211/1998. Dispõe sobre a atuação dos profissionais de enfermagem que trabalham com radiação ionizante. Rio de Janeiro: COFEN; 1998.

11.Marconi MA, Lakatos EM. Fundamentos de metodologia científica. 7a ed. São Paulo: Atlas; 2010.

12.Gil AC. Como elaborar projetos de pesquisa. $5^{\text {a }}$ ed. São Paulo: Atlas; 2010.

13.Ministério da Saúde (Br). Conselho Nacional de Saúde. Resolução nº66/12: diretrizes e normas regulamentadoras de pesquisa envolvendo seres humanos. Brasília (DF): Conselho Nacional de Saúde; 2012.

14.Instituto Nacional de Câncer José Alencar Gomes da Silva (Br). [site de internet]. Fatores de risco para o câncer. [citado em 29 abr 2014] Disponível em: http// www1.inca.gov.br/conteudo view.asp? $\mathrm{id}=322$.

15.Alvarenga LM, Ruiz MT, Bertelli ECP, Ruback MJC, Maniglia JV, Bertollo EMG. Avaliação epidemiológica de pacientes com câncer de cabeça e pescoço em um hospital universitário do noroeste do estado de São Paulo. Rev Bras Otorrinolaringol. 2008; 74:68-73.

16.Trindade BM, Campos RTP. Sistema computacional para dosimetria de nêutrons e fótons baseado em métodos estocásticos aplicado a radioterapia e radiologia. Radiol Bras. 2011; 44:109-16.

17.Ferreira NML, Souza MBB, Costa DB, Silva AC. Integridade cutâneo-mucosa: implicações para a família no cuidado domiciliário ao doente com câncer. Rev enferm UERJ. 2009; 17:246-51.

18.Smeltzer, SC, Bare, BG. Tratado de enfermagem médico-cirúrgica. $11^{\text {a }}$ ed. Rio de Janeiro: Guanabara Koogan; 2009.

19.Cuppari L. Nutrição clínica no adulto. $2^{a}$ ed. São Paulo: Manole; 2005.

20.Dealey C. Cuidando de feridas: um guia para as enfermeiras. $3^{\mathrm{a}}$ ed. São Paulo: Editora Atheneu; 2008.

21.Reis PED, Carvalho EC, Bueno PCP, Bastos JK. Aplicação clínica da Chamomilla recutita em flebites: estudo de curva. [citado em 29 abr 2014] Rev Latino-Am Enfermagem. 2011;19:[08 telas] Disponível em http:// 
www.redalyc.org/articulo.oa?id $=281421953002$.

22.Piper BF, Dibble SL, Dodd MJ, Weiss MC, Slaughter RE, Paul SM. The revised Piper fatigue scale: psychometric evaluation in women with breast cancer. Oncology Nursing Forum. 1998; 25 677-84.

23.Leite FMC, Ferreira FM, Cruz MAS, Lima EFA, Primo CC. Diagnósticos de enfermagem relacionados aos efeitos adversos da radioterapia. Rev Min Enferm. 2013; 17:940-5.

24.Luna NSA, Baeza MR, Castell EC, Santos FC, David HL, Castillo MMA. Intervención educativa: implementación de la agencia de autocuidado y adherencia terapéutica desde la perspectiva del paciente diabético. Rev enferm UERJ. 2013; 21:289-94.

25.Machado MMT, Leitão GCM, Holanda FUX. O conceito de ação comunicativa: uma contribuição para a consulta de enfermagem. Rev Latino-Am Enfermagem. 2005; [citado em: 29 abr 2014]13:723-8. Disponível em: http://www.scielo.br/scielo.php?script=sci arttext\&pid=S0104-11692005000500017\&lang = pt. 26.Cavalari E, Nogueira MS, Coelho SMLF, Cesarino CB, Martin JFV. Adesão ao tratamento: estudo entre portadores de hipertensão arterial em seguimento ambulatorial. Rev enferm UERJ. 2012; 20:67-72. 